\title{
Programas de prevención del abuso sexual en personas con discapacidad intelectual y del desarrollo: una revisión sistemática
}

\author{
Sexual abuse prevention programs for people with intellectual and \\ developmental disabilities: A systematic review
}

\begin{abstract}
Resumen
Las personas con discapacidad intelectual y del desarrollo, particularmente las mujeres, son especialmente vulnerables a las situaciones de abuso sexual. El objetivo principal de esta revisión es examinar los estudios empíricos sobre la efectividad de programas de prevención del abuso sexual en esta población. Una búsqueda sistemática en PsycINFO, Web of Science y Psicodoc, identificó diez estudios publicados sobre el tema. Los resultados obtenidos muestran que la mayoría de los programas de prevención están basados en la adquisición de habilidades conductuales y que hay otros centrados en la educación sexual. Aunque existen datos que demuestran la efectividad, sobre todo, de los programas de habilidades, la mayoría de los estudios no ofrecen resultados robustos con los que juzgar la efectividad de estas intervenciones. Las conclusiones de la revisión se centran en la necesidad de aumentar la investigación sobre la población con discapacidad intelectual y del desarrollo, especialmente en mujeres y en el ámbito del abuso sexual.
\end{abstract}

\section{Palabras clave}

Discapacidad intelectual y del desarrollo, abuso sexual, prevención, habilidades conductuales, educación sexual.

\begin{abstract}
People with intellectual and developmental disabilities, especially women, are particularly vulnerable to situations of sexual abuse. The aim of this review is to examine empirical studies on the effectiveness of sexual abuse prevention programs for this population. A systematic search on PsycINFO, Web of Science and Psicodoc identified ten published studies on the subject. The results obtained show that most prevention programs are based on the acquisition of behavioral abilities whereas others are based on sexual education. Although there are some data that demonstrate the effectiveness, particularly, of those programs based on abilities, the majority of studies do not provide robust evidence to judge the effectiveness of these interventions. The conclusions of the review are focused on the need to increase research about population with intellectual and developmental disabilities, especially women, and in the area of sexual abuse.
\end{abstract}

\section{Keywords}

Intellectual and developmental disabilities, sexual abuse, prevention, behavioural abilities, sexual education.

\section{Ma Icíar Martínez-Fortún López}

Universidad Complutense de Madrid. España

Clara Gesteira Santos

<clara.gesteira@ucm.es>

Universidad Complutense de Madrid. España

\section{Noelia Morán Rodríguez}

Universidad Complutense de Madrid. España

\section{María Paz García-Vera}

Universidad Complutense de Madrid. España

\section{Jesús Sanz Fernández}

Universidad Complutense de Madrid. España

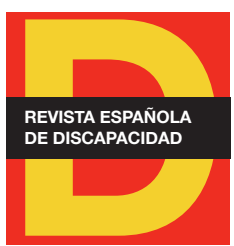

Para citar:

Martínez-Fortún, M. I. et al. (2021).

Programas de prevención del abuso sexual en personas con discapacidad intelectual y del desarrollo: una revisión sistemática. Revista Española de Discapacidad, 9(1), pp. 75-100.

Doi: <https://doi.org/10.5569/23405104.09.01.04>

Fecha de recepción: 19-10-2020 Fecha de aceptación: 18-05-2021 


\section{Introducción}

La discapacidad intelectual, según el DSM-5 (American Psychiatric Association-APA, 2014), es un trastorno que comienza durante el período del desarrollo y que incluye tanto limitaciones del funcionamiento intelectual como del comportamiento adaptativo en los dominios conceptual, social y práctico. Dependiendo de la afectación de estos dominios se puede hablar de diferentes grados de discapacidad intelectual: leve, moderado, grave o profundo. En ocasiones, no se puede valorar el grado de discapacidad intelectual debido a deterioros sensoriales o físicos asociados, como la ceguera o sordera, problemas en el comportamiento o discapacidad locomotora (APA, 2014). Aunque discapacidad intelectual es la etiqueta diagnóstica, en realidad, el término que se suele utilizar para referirse a esta población es discapacidad intelectual y del desarrollo ${ }^{1}$.

Habitualmente es difícil encontrar una cifra exacta sobre la prevalencia de la discapacidad intelectual y del desarrollo en estudios epidemiológicos, dado que este tipo de discapacidad suele aparecer unida a otras (por ejemplo, discapacidad física). Sin embargo, la última actualización de la base estatal de datos con valoración del grado de discapacidad muestra que un 6,6\% de la población de España tiene algún tipo de discapacidad y de ese porcentaje, el 8,7 \% son personas que tienen discapacidad intelectual y del desarroIlo (Subdirección General de Planificación, Ordenación y Evaluación, 2018).

Por otro lado, aunque tradicionalmente se ha dado mucha importancia al aspecto intelectual de la discapacidad intelectual y del desarrollo, hoy en día cobra mayor relevancia el aspecto funcional, que hace referencia a la capacidad de las personas para adaptarse a la sociedad a nivel personal, social y laboral. Este énfasis en el contexto social haría también difícil diferenciar la discapacidad intelectual y del desarrollo de otros tipos de discapacidad, como la discapacidad psicosocial (Drew et al., 2011), puesto que en ambos casos se subrayaría el estigma, la discriminación y la exclusión a los que se ven sometidas estas personas. No obstante, en el caso de la discapacidad psicosocial esto ocurriría como consecuencia de padecer trastornos mentales (por ejemplo, trastornos afectivos, de ansiedad o psicóticos), así como por el uso de los servicios de salud mental.

En todo caso, teniendo en cuenta esta idea de que la discapacidad se hace patente en el encuentro de la persona con la sociedad (Recio et al., 2013), muchos de los problemas que sufren las personas con discapacidad intelectual y del desarrollo no están en sus manos, sino que vienen causados por agentes externos. Uno de ellos es el abuso sexual (Doughty y Kane, 2010).

\section{Abuso sexual y discapacidad intelectual y del desarrollo: definición, tipos y prevalencia}

Desde el punto de vista legal, en nuestro país, se entiende por abuso sexual cualquier forma de atentar contra la libertad sexual de otra persona, sin violencia o intimidación y sin consentimiento, mientras que

1. En adelante, en el texto, se utilizará el término discapacidad intelectual y del desarrollo, a pesar de que en algunos estudios se refieren únicamente a discapacidad intelectual o discapacidad del desarrollo. 
la definición de agresión sexual contempla violencia e intimidación al atentar contra la libertad sexual de otra persona (España. Ley Orgánica 10/1995, de 23 de noviembre, del Código Penal). Sin embargo, en la literatura científica sobre abuso y discapacidad intelectual y del desarrollo no se suele hacer una distinción entre estos dos términos (abuso y agresión sexual), ya que las personas con discapacidad intelectual y del desarrollo no suelen defenderse ante una situación en la que se atente contra su libertad sexual, por miedo o por desconocimiento (Sobsey y Varnhagen, 1988), por lo que no se suelen encontrar signos de violencia. De hecho, en la literatura científica tradicional (por ejemplo, Brown y Turk, 1992; Finkelhor et al., 1990) se utiliza el término abuso sexual de manera general, para referirse a los actos sexuales que son llevados a cabo sobre alguien que no quiere o no es capaz de consentir esos actos. De la misma manera, McCarthy (1993) lo define como cualquier contacto sexual que no es deseado por una parte y que es para la satisfacción de la otra parte.

Asimismo, se pueden diferenciar dos tipos de abuso: abuso sexual con contacto, que puede incluir masturbaciones, tocamientos y penetración, o abuso sexual sin contacto, que incluye acoso, pornografía e insinuaciones (Verdugo et al., 2002). En un estudio realizado por Stromness (1993), se indica que el primero parece mucho más frecuente, ya que el $71 \%$ de las participantes (mujeres con discapacidad intelectual y del desarrollo) habían sufrido abuso directo, es decir, con contacto, mientras que el $29 \%$ restante, habían sido víctimas de abuso sin contacto.

Es difícil encontrar estudios que aporten datos definitivos sobre el porcentaje real de personas con discapacidad intelectual y del desarrollo que sufren abuso sexual ya que, tal y como se mencionaba anteriormente, normalmente se mezcla a las personas con discapacidad intelectual y del desarrollo con las que tienen otros tipos de discapacidad. Estudios que intentan hallar la proporción de personas con discapacidad intelectual y del desarrollo que han sufrido abuso sexual en algún momento de su vida, muestran porcentajes que varían entre el $8 \%$ en el Reino Unido al $58 \%$ en Estados Unidos (Bruder y Kroese, 2005). Además, estudios comparativos entre población con y sin discapacidad muestran que las personas con discapacidad intelectual y del desarrollo suelen tener más probabilidades de sufrir abuso sexual que aquellas sin discapacidad (Euser et al., 2015; Lin et al., 2009; Martin et al., 2006; Reiter et al., 2007; Spencer et al., 2005; Withers y Morris, 2012), por lo que parece que tendrían una vulnerabilidad mayor en estos casos.

\section{Vulnerabilidad de las personas con discapacidad intelectual y del desarrollo a sufrir abuso sexual: causas generales e interseccionalidad en las mujeres}

Entre las causas por las que las personas con discapacidad intelectual y del desarrollo podrían ser más vulnerables a sufrir abuso sexual encontramos, por un lado, la dificultad que tienen para diferenciar entre lo que es apropiado y lo que no (Martinello, 2014), es decir, pueden confundir una situación de abuso con la atención de los cuidadores o cuidadoras. Este problema puede ser debido a la pobre educación sexual que reciben, por los mitos asociados a esta población, como la creencia de que las personas con discapacidad intelectual y del desarrollo carecen de sexualidad (Alemany et al., 2012). Esto lleva a no tener una identidad sexual desarrollada y a la ausencia de un modelo de rol sexual, sobre todo, en personas institucionalizadas (Verdugo et al., 2002). Por otro lado, existe un mayor riesgo de abuso por la alta deseabilidad social presente 
en esta población, que lleva a estas personas a buscar y demandar atención, aunque esta suponga una situación de abuso sexual (Recio et al., 2013). Además, su alta dependencia y la falta de intimidad que esto supone, coloca a estas personas en una situación de vulnerabilidad muy elevada, ya que las convierte en fácilmente manipulables (Nosek et al., 2001). Por último, la falta de conocimiento sobre cómo defenderse ante un abuso sexual (Sobsey y Varnhagen, 1988), la ausencia de competencia social y la escasa capacidad para juzgar e informar son otros de los factores que influyen en que las personas con discapacidad intelectual y del desarrollo sufran abuso sexual (Chodan et al., 2017). Algunos de los factores mencionados anteriormente pueden ser la causa de que la mayoría de los abusos sexuales se produzcan por personas que conocen a las víctimas, cuidadores o cuidadoras, familiares o personas conocidas. De hecho, el perfil de quien abusa suele responder al de un hombre que, en la mayoría de los casos, es conocido por sus víctimas (Bruder y Kroese, 2005).

Sin embargo, existen otros factores de riesgo ajenos a la condición de discapacidad, que tienen más que ver con el propio abuso sexual y su ligazón con la violencia hacia las mujeres. Al igual que ocurre para la población general, el abuso sexual en las personas con discapacidad intelectual y del desarrollo parece ser también un fenómeno con resultado feminizado, que se da con mucha más frecuencia en mujeres que en hombres. Así, se estima que entre un 39 y $68 \%$ de las mujeres con discapacidad intelectual y del desarrollo, frente a un 16 y $30 \%$ de los hombres, son abusadas antes de cumplir los 18 años (Martinello, 2014). Couwenhoven (2013) afirma que, entre la población adulta, el $83 \%$ de las mujeres sufriría abusos sexuales frente a un porcentaje inferior de hombres (32\%). Otros estudios más recientes corroboran también este hecho. Giménez-García et al. (2017), por ejemplo, afirman que el $30 \%$ de las mujeres participantes del estudio referían haber sido víctimas de abuso sexual, frente a un 13,2\% de hombres y Morell-Mengual et al. (2016) encuentran datos significativamente mayores de abuso en mujeres que en hombres. Entre la muestra que analizan, el $17 \%$ de las mujeres han sido abusadas en comparación con el $5 \%$ de los hombres.

En cualquier caso, es importante puntualizar que ni la condición de discapacidad intelectual y del desarrollo ni la de mujer son factores de vulnerabilidad per se, sino que son el entorno y la exposición a situaciones de discriminación por parte de la sociedad los que incrementan dicha vulnerabilidad (Gomiz, 2016). En este sentido, las mujeres con discapacidad sufren lo que se conoce como "discriminación interseccional" o doble discriminación, al poseer más de una identidad considerada inferior por la sociedad (Cavalcante, 2018; Comité Español de Representantes de Personas con Discapacidad, CERMI, 2013; Gomiz, 2016; Viñuela, 2009). Es decir, están sometidas al mismo tiempo a la discriminación que sufren las mujeres y a la discriminación que sufren las personas con discapacidad, lo que las coloca en una situación de mayor vulnerabilidad. De manera coherente, datos de la macroencuesta realizada por la Fundación CERMI Mujeres en 2015 a 10.171 mujeres, de entre las cuales 442 tenían discapacidad, muestran que un 8,5\% de las mujeres con discapacidad declaró haber sufrido violencia sexual por su anterior pareja, en comparación con un 3,9\% de las mujeres sin discapacidad que habían sufrido violencia. De manera más general, el 35,1\% de las mujeres con discapacidad declaró haber sufrido algún tipo de violencia sexual y física en algún momento de su vida por su pareja, expareja o persona desconocida, frente al $23,7 \%$ de las mujeres sin discapacidad que afirmaron lo mismo (Cavalcante, 2018). 


\section{Consecuencias psicológicas y sociales del abuso sexual en discapacidad intelectual y del desarrollo}

Sufrir abuso sexual causa en las personas con discapacidad intelectual y del desarrollo un alto estrés, que puede desencadenar una vivencia traumática. La manifestación de estas vivencias en las personas con discapacidad intelectual y del desarrollo se puede dar de muchas formas: ansiedad, depresión, cambios conductuales o trastorno de estrés post-traumático (TEPT), entre otros (Recio et al., 2013). Brackenridge y Morrissey (2010), realizaron un estudio en el que el $15 \%$ de las personas con discapacidad intelectual y del desarrollo habían desarrollado síntomas de TEPT a lo largo de su vida tras un abuso sexual. De ellas, algunas tenían, además, un diagnóstico de trastorno del estado de ánimo, otras, trastornos de personalidad y otras, trastornos de ansiedad. Sufrir abuso sexual también produce, en más del $95 \%$ de las personas con discapacidad intelectual y del desarrollo que lo han vivido, retraimiento social, sentimientos de culpa, conductas de agresión hacia otras personas, menos confianza en sí mismas y problemas de conducta (Chodan et al., 2017). Estos trastornos, además, según Wigham et al. (2011), se manifestarían de diferente manera según el nivel de desarrollo de cada persona. Así, por ejemplo, en el caso de las personas que tienen discapacidad intelectual y del desarrollo con habilidades de comunicación y expresión verbal reducidas, las reacciones ante eventos traumáticos como el abuso solo podrían ser observadas por cambios en el comportamiento.

Además de todo lo anterior, las personas con discapacidad intelectual y del desarrollo que sufren abuso deben enfrentarse después a muchas dificultades, por ejemplo, en el ámbito judicial. En torno a ellas hay algunos mitos relacionados con su incapacidad para declarar. Entre estos mitos existe la idea de que tienden a fantasear sobre lo que viven, además de que se piensa que no sufren por ser víctimas de abuso (Alemany et al., 2012). Esto hace que no se las crea o que no tengan la oportunidad de expresarse o defenderse, contribuyendo a crear situaciones de victimización secundaria o revictimización, o lo que es lo mismo, situaciones estresantes a las que son sometidas las víctimas tras haber sufrido un delito, que surgen de la relación con los y las profesionales de los servicios sanitarios, policiales o judiciales, y que les afectan de manera grave y traumática, generando importantes consecuencias psicológicas y emocionales (Gutiérrez de Piñeres et al., 2009).

\section{Programas de prevención del abuso sexual en discapacidad intelectual y del desarrollo: estado de la cuestión y objetivos de la revisión}

Ante el elevado porcentaje de personas con discapacidad intelectual y del desarrollo que son víctimas de abuso sexual y los problemas psicológicos y sociales que pueden derivarse de estas situaciones, no son pocos los intentos que se han hecho para desarrollar programas de prevención cuyo fin sea reducir el riesgo específico de abuso entre esta población.

Los programas de prevención del abuso sexual en personas con discapacidad intelectual y del desarrollo tradicionalmente promocionan, por un lado, la educación sexual, el conocimiento del propio cuerpo y la edu- 
cación de relaciones adecuadas (Kim, 2016) y por el otro, el desarrollo de habilidades para afrontar y prevenir el abuso sexual (Lumley et al., 1998). En resumen, los programas de prevención se dirigen a intervenir sobre los factores de vulnerabilidad de las personas con discapacidad intelectual y del desarrollo tratando, de esta manera, de disminuir el riesgo de ser víctimas de abuso sexual y de paliar los efectos derivados de los abusos.

Se han encontrado 5 revisiones (Barger et al., 2009; Bruder y Kroese, 2005; Chrastina y Vecerova, 2018; Doughty y Kane, 2010; Mikton et al., 2014) específicamente dirigidas al análisis de los programas de prevención en esta población, pero de ellas, solo 3 son sistemáticas (Barger et al., 2009; Doughty y Kane, 2010; Mikton et al., 2014). Barger et al. (2009) encontraron 5 artículos que se centraban únicamente en los abusos sexuales en mujeres con discapacidad intelectual y del desarrollo. Una de las conclusiones de esta revisión fue que las mujeres con discapacidad intelectual y del desarrollo tenían un mayor riesgo de victimización por abuso sexual. Sin embargo, no encontraron recursos destinados a estudiar la prevención de abuso sexual para mujeres con discapacidad intelectual y del desarrollo y, por tanto, resaltaron la acuciante necesidad de continuar trabajando para desarrollar programas de prevención de abuso sexual para personas con discapacidad intelectual y del desarrollo. Doughty y Kane (2010), revisaron 6 estudios sobre programas de prevención basados en habilidades de autoprotección, de los cuales concluyeron que las habilidades aprendidas en los programas que se habían revisado se mantuvieron y generalizaron en el tiempo en cierta medida, si bien el periodo de seguimiento se extendía tan solo entre una semana y tres meses. Por otro lado, detectaron una mayor tendencia a trabajar sobre las habilidades de protección en los estudios más actuales recogidos en la revisión. Por último, Mikton et al. (2014) revisaron 10 estudios en los que se incluían discapacidades tanto mentales como físicas y todas las formas de violencia. Concluyeron que habían revisado pocos artículos y calificaron todos los estudios como débiles ya que no habían utilizado medidas de violencia real como resultado ni habían proporcionado datos estadísticos de la muestra.

A pesar de que estas revisiones aportan datos útiles, siguen siendo escasas y, además, están desactualizadas. Todas ellas revisan un número reducido de estudios y, o bien se centran en grupos muy concretos dentro de la población con discapacidad intelectual y del desarrollo o son demasiado generales. Además, las revisiones de Barger et al. (2009) y Doughty y Kane (2010), solo analizan los resultados de los estudios de manera narrativa, sin hacer referencia a datos numéricos.

Teniendo en cuenta las limitaciones de estas revisiones y la importancia que tienen sobre las personas con discapacidad intelectual y del desarrollo los programas de prevención del abuso, el objetivo de la presente revisión es hacer una recopilación y análisis sistemático y actualizado de los estudios empíricos sobre programas de prevención que se llevan a cabo para reducir el riesgo de que las personas con discapacidad intelectual y del desarrollo sean víctimas de abuso sexual.

La pregunta concreta que se tratará de responder mediante esta revisión sistemática es cuál es la efectividad de los programas de prevención del abuso sexual aplicados a personas con discapacidad intelectual y del desarrollo, en términos de respuesta a las distintas intervenciones (por ejemplo, habilidades adquiridas de prevención del abuso y nivel de conocimiento sobre sexualidad). Asimismo, se tratará de establecer cuál de las dos formas tradicionales de prevención (la educación sexual o el entrenamiento en habilidades) es la más efectiva y, por tanto, la que debería considerarse tratamiento de elección en estos casos. En base a la evidencia disponible en las revisiones examinadas previamente (por ejemplo, Doughty y Kane, 2010), se hipotetiza que los programas de habilidades ofrecerán mejores resultados que los de educación sexual, aunque seguramente, y tal y como sucedía en otros trabajos previos en el campo de la discapacidad intelectual y del desarrollo, la evidencia disponible no será suficientemente robusta como para sacar conclusiones contundentes. 


\section{Método}

En este estudio de revisión se siguieron las indicaciones propuestas por el grupo PRISMA (Moher et al., 2009) para búsquedas sistemáticas. En primer lugar, se utilizaron las bases PsycINFO y Web of Science, con la siguiente combinación de palabras en cualquier campo: ("mental disability" OR "mental retardation") AND ("rape" OR "sexual assault" OR "sexual abuse") AND ("prevention program" OR "intervention" OR "therapy"). En segundo lugar, se hizo uso de la base de datos electrónica Psicodoc con la combinación de palabras en cualquier campo: ("discapacidad intelectual") AND ("abuso sexual") AND ("programas de prevención" OR "intervención"). La búsqueda se realizó en abril de 2020, incluyendo los artículos publicados en todas las fechas, pero limitando la búsqueda a los resultados publicados en revistas científicas. Dada la escasez de artículos encontrados en revisiones previas, no se establecieron filtros por idioma, características sociodemográficas de la población, ni en función de criterios metodológicos (por ejemplo, que los estudios incluyeran un grupo de control).

Las búsquedas anteriores permitieron identificar 224 publicaciones y, tras descartar 3 artículos que aparecían en dos o más bases de datos bibliográficas y añadir 6 publicaciones diferentes identificadas tras consultar la bibliografía citada en los estudios consultados, se obtuvieron 227 publicaciones con fechas comprendidas entre 1998 y 2020. En la figura 1 se presenta, en forma de diagrama de flujo, el proceso de identificación, cribado y selección de los estudios sobre programas de prevención del abuso sexual en personas con discapacidad intelectual y del desarrollo.

En función de los objetivos del presente estudio y sobre la base de la lectura de sus títulos, datos bibliográficos básicos y resúmenes, se cribaron las 227 publicaciones inicialmente encontradas, en función de si cumplían con los siguientes criterios de inclusión: a) estudios empíricos; b) que examinaran muestras de personas con discapacidad intelectual y del desarrollo; c) que incluyeran programas específicos de prevención del abuso sexual para estas personas y sus resultados (cualitativos o cuantitativos). Así, en esta fase se excluyeron 123 documentos. Concretamente, tal y como se aprecia en la figura 1, 101 se descartaron porque no tenían que ver con el tema del estudio, 5 eran revisiones, 3 no eran estudios empíricos, 7 estaban enfocados en otro tipo de población y 7 no incluían programas o tratamientos específicos ni sus resultados.

De las 104 publicaciones resultantes de la fase de cribado, se obtuvo el texto completo de 22 (82 publicaciones fueron descartadas por no disponer de acceso al texto completo) y, en función de su lectura, se volvieron a evaluar esas publicaciones en cuanto al cumplimiento de los criterios de inclusión. Tal y como se refleja en la figura 1, en esta fase se excluyeron 12 publicaciones, 3 de ellas porque, aunque incluían propuestas de programas o estrategias de prevención específica e incluso protocolos de estudio completos, no incluían la evaluación (cualitativa o cuantitativa) de sus resultados y por tanto, no podían considerarse estudios empíricos, y otras 9 que sí constituían estudios empíricos sobre programas de prevención referidos a personas con discapacidad intelectual y del desarrollo pero en este caso, eran estas personas las que habían abusado sexualmente en lugar de ser las víctimas del abuso sexual.

Finalmente, tras el proceso de cribado y elegibilidad de las publicaciones inicialmente identificadas, en la presente revisión sistemática se incluyeron 10 publicaciones que informaban de 9 estudios diferentes, sobre los cuales se realizó una síntesis cualitativa. 
Tanto el proceso de búsqueda, como el de selección y síntesis de las publicaciones relevantes para esta revisión, fueron llevados a cabo por dos investigadoras, una primera implicada en la totalidad del proceso, y una segunda, que actuó como supervisora de la anterior. Los casos en los que existía duda eran resueltos por consenso entre ambas revisoras.

Figura 1. Diagrama de flujo del proceso de búsqueda y selección de los estudios sobre los programas de prevención del abuso sexual en personas con discapacidad intelectual y del desarrollo
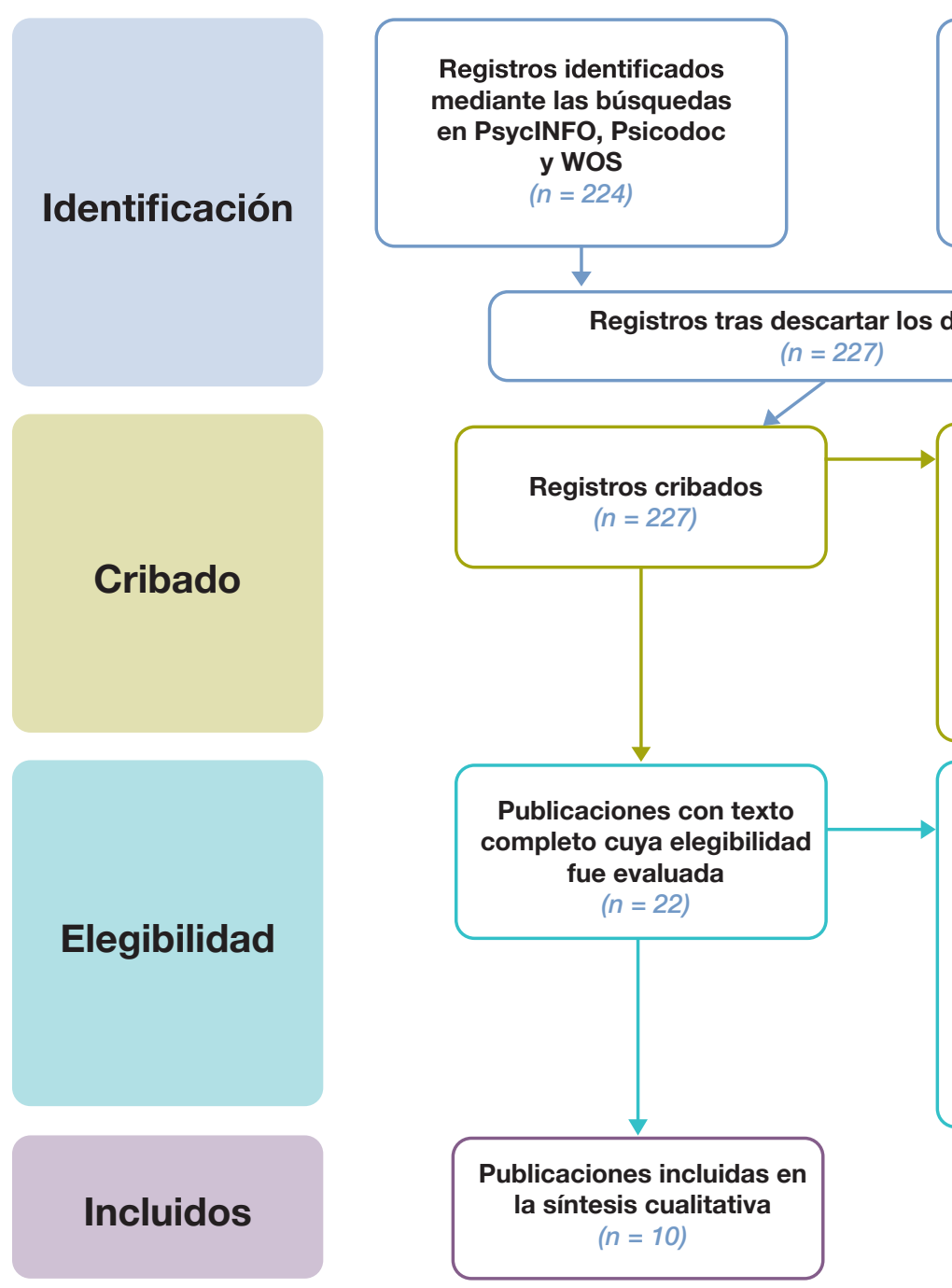

Registros identificados por la bibliografía citada

en las publicaciones consultadas $(n=6)$

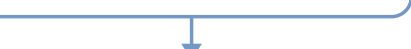

duplicados

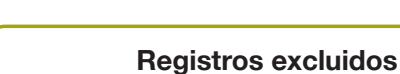

Tema diferente: $(n=101)$

- Revisión/trabajo teórico: $(n=5)$

- Estudios no empíricos: $(n=3)$

- Muestra de personas sin DID: $(n=7)$

- Estudios empíricos que no incluyen programas de tratamiento específicos: $(n=7)$

Fuente: elaboración propia (basada en Moher et al., 2009). 


\section{Resultados}

En la tabla 1 se muestran las principales características y los resultados de los 10 artículos seleccionados para esta revisión. Entre los programas de prevención que aparecen en estos artículos seleccionados, se pueden diferenciar dos tipos: los que están encaminados a la adquisición de habilidades conductuales y los que se centran en la prevención del abuso sexual a través de la educación sexual y el conocimiento sobre lo que es abuso. Los programas que se centran en el aprendizaje de habilidades para la prevención del abuso son más completos y complejos, ya que antes de enseñar y practicar las habilidades conductuales es necesario dotar de conocimientos tanto de sexualidad y relaciones sexuales como de abuso sexual. Por esto, aunque en esta revisión se diferencia entre dos tipos de artículos, es importante recalcar que los programas de habilidades en realidad incluyen a los otros, puesto que también incluyen psicoeducación sobre abuso sexual. Entre los 10 artículos analizados, hay 8 (Dryden et al., 2014, 2016; Egemo-Helm et al., 2007; Graff et al., 2018; Hickson et al., 2015; Kim, 2016; Lumley et al.,1998; Miltenberger et al.,1999) cuyo objetivo es la evaluación de un programa que se centra en la adquisición de habilidades de prevención; mientras que los 2 restantes (Bowman et al., 2010; Lund y Hammond, 2014), son programas de educación sexual.

\subsection{Programas de adquisición de habilidades conductuales}

Los 8 artículos que se basan en la adquisición de habilidades conductuales se pueden dividir a su vez, en 2 grupos. En el primer grupo se encuentran 4 programas que están basados en un mismo protocolo de entrenamiento (Egemo-Helm et al., 2007; Kim, 2016; Lumley et al., 1998; Miltenberger et al., 1999), concretamente, en el protocolo de entrenamiento propuesto por Lumley et al. (1998); el segundo grupo está formado por 4 artículos que se basan en otros tipos de entrenamiento centrados en juegos de rol, combinados con otras formas de entrenamiento (Dryden et al., 2014, 2016; Graff et al., 2018; Hickson et al., 2015).

\subsubsection{Programas basados en el protocolo de entrenamiento de Lumley et al. (1998)}

Estos 4 artículos (Egemo-Helm et al., 2007; Kim, 2016; Lumley et al., 1998; Miltenberger et al., 1999) están basados en un entrenamiento diseñado por Lumley et al. (1998). Dicho entrenamiento se centra en el aprendizaje de habilidades para identificar, rechazar (NO), abandonar (VAYA) e informar (DIGA) de una situación de abuso sexual. El protocolo comienza con explicaciones sobre el abuso sexual, las partes del cuerpo, las relaciones apropiadas e inapropiadas y los comportamientos sexuales adecuados e inadecuados. En todos los casos las habilidades aprendidas se ensayan con juegos de rol, en los que se plantea una situación simulada de abuso desempeñada por un entrenador del programa, en este caso, un hombre.

No obstante, es importante tener en cuenta que, aunque todos los estudios de este grupo siguen estos mismos principios, existe cierta variabilidad dentro de ellos, por ejemplo, en el número de sesiones, que se sitúan entre 3 (Egemo-Helm et al., 2007) y 10 (Miltenberger et al., 1999). Además, Kim (2016) adapta este programa a niñas pequeñas y hace sesiones más cortas, de 30 minutos en lugar de una hora, y utiliza posters, juegos de tarjetas y cuentos para colorear. 
En estos cuatro estudios la muestra está compuesta exclusivamente por mujeres con discapacidad intelectual y del desarrollo leve-moderada, en grupos reducidos (Egemo-Helm et al., 2007 n= 4; Kim, $2016 n=3$; Lumley et al., $1998 n$ = 6; y Miltenberger et al.,1999 $n=5$ ), lo cual limita el uso de estadísticos de comparación de los resultados obtenidos.

Aunque la manera tradicional de evaluar programas de prevención de abuso sexual en personas con discapacidad intelectual y del desarrollo suele ser mediante métodos indirectos, es decir, mediante la medición del conocimiento o descripción verbal de lo que harían las participantes ante una supuesta situación de abuso sexual, los programas que siguen la línea de Lumley et al. (1998) añaden métodos más directos de evaluación. Por ello, en los cuatro estudios de este grupo, tanto antes como después del entrenamiento, no solo se evalúa el conocimiento teórico sobre el tema, sino también las habilidades directamente expresadas en un escenario ficticio de abuso sexual, lo cual se consideraría un método más directo. En todos los programas de este grupo, de hecho, se hace una evaluación previa del conocimiento sexual y las reacciones de las participantes, utilizando juegos de rol, en un escenario donde se simula una situación de abuso sexual desempeñada por un entrenador (masculino) del programa y donde la participante es consciente de que está siendo evaluada. También se utiliza la evaluación in situ ante una situación de abuso que la participante desconoce y en la que no es consciente de que se la está evaluando. Esta evaluación se repite después del entrenamiento para comparar los resultados, obteniendo de esta forma una evaluación pre y otra, post entrenamiento.

Según el método de Lumley et al. (1998) la evaluación de la conducta se realiza calificando las respuestas de las participantes en una escala de 4 puntos, registrando 1 punto para cada uno de los comportamientos objetivo. Estos comportamientos se definen como: no acepta participar de la solicitud que se le plantea, se niega verbalmente ante la situación, abandona la situación o pide al solicitante que se vaya e informa de lo ocurrido a un miembro del equipo que se encarga del entrenamiento.

En los cuatro estudios mencionados de este grupo los resultados que se obtienen reflejan cierta efectividad del tratamiento, aunque solamente con datos descriptivos. Por un lado, Egemo-Helm et al. (2007) y Kim (2016), aportan puntuaciones individuales de cada participante, que, en su mayoría, obtienen en la evaluación post-intervención, tanto en juegos de rol como en evaluación in situ, la máxima puntuación posible (4 puntos). Sin embargo, estos resultados son solamente individuales y descriptivos y no se utiliza ningún estadístico que muestre si existen diferencias significativas pre-post. Por otro lado, en los programas de Lumley et al. (1998) y Miltenberger et al. (1999), las puntuaciones reflejan las medias de todas las participantes, siendo las puntuaciones post-entrenamiento más elevadas que las obtenidas en evaluaciones previas al entrenamiento, tanto en evaluaciones mediante juegos de rol, como en las evaluaciones in situ. En ambos casos, la puntuación que se obtiene en la evaluación post entrenamiento mediante juegos de rol es la máxima posible $(M=4)$, mientras que en el caso de la evaluación in situ, Miltenberger et al. (1999) muestran una puntuación más alta (pre: $M=0,6$; post: $M=3,6$ ) que la que obtienen Lumley et al. (1998) (pre $M=1,3$; post $M=1,8)$. Esto podría ser debido a que el entrenamiento de Miltenberger et al. (1999) tiene una duración de 10 sesiones, mientras que el de Lumley et al. (1998) está compuesto por tan solo 5 sesiones. Tal y como ocurre con los estudios anteriores, ni Lumley et al. (1998) ni Miltenberg et al. (1999) especifican ninguna medida de comparación estadística pre-post, solamente muestran los resultados de las medias antes y después.

Los cuatro programas reflejan evaluaciones de seguimiento a largo plazo, con el fin de observar si las habilidades aprendidas en el momento del entrenamiento se mantienen en el tiempo. En los casos de Mil- 
tenberger et al. (1999) y Lumley et al. (1998), el seguimiento se realiza 1 mes después de haber completado el entrenamiento. En las medidas de juegos de rol se observan puntuaciones altas $(M=4$ y $M=3,6)$ en ambos estudios, por el contrario, en las medidas de evaluación in situ solo se observan puntuaciones altas en el caso de Milteberger et al. (1999) ( $M=3$ ). En los programas de Egemo-Helm et al. (2007) y Kim (2016), el seguimiento se realiza aproximadamente 3 meses después de acabar el entrenamiento y casi todas las participantes obtienen la máxima puntuación, con mejores puntuaciones las participantes del programa de Kim (2016). No obstante, igual que ocurre en el post-entrenamiento, siguen siendo datos descriptivos, sin medidas estadísticas que corroboren las diferencias significativas en los seguimientos.

\subsubsection{Otros tipos de entrenamiento}

De los 8 artículos que enseñan protocolos en los que se trabajan habilidades conductuales, otros 4 (Dryden et al., 2014, 2016; Graff et al., 2018; Hickson et al., 2015) utilizan otras formas de entrenamiento en las que se utilizan siempre juegos de rol que, en algunos casos, se combinan con otras formas de intervención. Aunque son 4 los artículos publicados, es importante reseñar que se trata solamente de 3 estudios, puesto que los 2 trabajos presentados por Dryden et al. $(2014,2016)$ pertenecen en realidad a una misma investigación, siendo la publicación de 2014 la que presenta los resultados post intervención y la de 2016 la que muestra los resultados de seguimiento.

En cuanto a los protocolos en sí, Graff et al. (2018), utilizan los juegos de rol combinados con actividades de toma de decisiones y cuestionarios basados en el programa de "Opciones Positivas". Este programa consiste en enseñar habilidades sobre sexualidad y relaciones saludables mediante un temario dividido en cinco partes: relaciones y autoconciencia, maduración, ciclo de vida, salud sexual y ser fuerte y mantenerse a salvo. Las habilidades se enseñan mediante el uso de juegos de rol, libros de trabajo, hojas de actividades e imágenes coloridas para el grupo de intervención. Otros instrumentos utilizados en el estudio de Hickson et al. (2015) para la adquisición de habilidades de prevención de abuso son las viñetas que reflejan situaciones de abuso sexual, presentadas a lo largo de las 12 sesiones de instrucción siguiendo el plan de estudios de Khemka y Hickson (2008) conocido como ESCAPE DD. En primer lugar, se enseñan los conceptos básicos y los objetivos de autoprotección, y después se proponen modelos de toma de decisiones y se proporciona entrenamiento para evitar situaciones de abuso mediante la identificación del problema, generación de alternativas, evaluación de estas y selección del mejor curso de acción para la situación. Por último, Dryden et al. (2014, 2016), utilizan simulaciones de la vida real en diversas situaciones con contenidos muy diversos como, por ejemplo, escenarios que involucran a una persona que acosa, enfrentarse a rumores que se están difundiendo, establecer límites, etc.

En este segundo grupo de estudios, que utilizan, además de los juegos de rol, otras formas de entrenamiento, el número de participantes es más elevado que en el primer grupo de estudios basados en el entrenamiento de Lumley et al. (1998). Así, en casi todos estos programas participan alrededor de 50 personas.

En estos trabajos, además, se evalúan los conocimientos y habilidades de dos grupos, un grupo de intervención y un grupo control. Sin embargo, la manera de asignación de los participantes a cada uno de los grupos es diferente en cada caso. En el estudio de Hickson et al. (2015), la asignación a cada grupo es de forma aleatoria. En el caso de Graff et al. (2018), la asignación a los grupos no es aleatoria sino de conveniencia, en base de la pertenencia de los y las participantes a un curso o a otro. El estudio de Dryden et al. $(2014,2016)$ utiliza un diseño cuasi-experimental pero no especifica la forma en la que establece los grupos. 
En todos los estudios se toman medidas pre y post intervención y en el caso de Dryden et al. (2014) y el de Hickson et al. (2015), además, medidas de comparación entre el grupo de tratamiento y el grupo control en el post-tratamiento. Sin embargo, es importante precisar que las medidas tomadas en cada estudio son diferentes entre sí. Graff et al. (2018) evalúan mediante juegos de rol combinados con evaluación in situ y actividades de toma de decisiones. También Hickson et al. (2015) utilizan escalas de toma de decisiones, así como preguntas sobre lo que está ocurriendo y lo que debería ocurrir en viñetas que representan situaciones de abuso. Por último, Dryden et al. (2014, 2016), evalúan mediante un cuestionario con diferentes preguntas sobre conocimiento, confianza para protegerse y conducta de autodefensa y seguridad, que son los temas tratados durante el entrenamiento.

En cuanto a los resultados, en la comparación entre el grupo de intervención y grupo control, Dryden et al. (2014) encuentran diferencias estadísticamente significativas en el post tratamiento en favor del grupo de intervención, en concreto, en las escalas de conocimiento, seguridad y autodefensa, confianza en defensa personal y seguridad al decir no $(p<0,05)$. Además, el grupo de intervención en el post entrenamiento obtiene mejoras significativas en las puntuaciones en casi todas las preguntas del cuestionario $(p<0,05)$. De la misma manera, Hickson et al. (2015) encuentran diferencias significativas entre el grupo de intervención y el grupo control en el post entrenamiento, en favor del primero en la subescala "toma de decisiones efectiva en general" $(F(1,52)=6,61 ; p=0,013 ; \eta 2=0,113)$ y en la subescala "toma de decisiones efectiva y segura" $(F(1,52)=$ $5,73 ; p=0,02 ; \eta 2=0,099)$ y, además, muestran mejoras significativas de las puntuaciones en la variable principal "toma de decisiones general" del grupo de intervención en el post entrenamiento respecto al pre ( $t$ (29) $=4,27 ; p=0,001)$. En el estudio restante solamente se hacen comparaciones pre-post. Así, Graff et al. (2018), comparan las puntuaciones antes y después del entrenamiento, obteniendo el grupo de intervención mejoras estadísticamente significativas en el post respecto al preentrenamiento $(p<0,01)$, no así el grupo control.

De este grupo de 4 estudios, solamente hay uno en que se hicieran seguimientos de los participantes, el de Dryden et al. (2016) que es, como ya se ha mencionado, un segundo estudio realizado sobre el original (Dryden et al., 2014). En este seguimiento, realizado un año después, se encuentran en el grupo de intervención mejores puntuaciones en 3 de las escalas que utilizan para evaluar sobre la autodefensa y seguridad, con diferencias estadísticamente significativas $(p<0,05)$.

\subsection{Programas de educación sexual}

Por otro lado, además de los 8 estudios que tienen que ver con la adquisición de habilidades conductuales, hay 2 que se centran en el conocimiento del abuso y la educación sexual a través de talleres como forma de prevención del abuso sexual (Bowman et al., 2015; Lund y Hammond, 2014). En el primero de ellos (Bowman et al., 2015) se desarrollan talleres dirigidos al personal que trabaja con personas con discapacidad del desarrollo, para dotarles de conocimientos y de las estrategias necesarias para detectar y prevenir el abuso sexual en esta población y en el segundo, se propone un plan de estudios (Lund y Hammond, 2014).

\subsubsection{Talleres dirigidos al personal}

En el caso de Bowman et al. (2010), en el programa participan 124 mujeres jóvenes que trabajan con esta población. El taller tiene una duración de 4 horas en las que se explican contenidos relacionados con las 
leyes de abuso sexual, los factores de riesgo de las personas con discapacidad, signos físicos y conductuales del abuso, cómo enseñar educación sexual, etc. Para medir la efectividad del taller, se administra un cuestionario antes y después del entrenamiento relacionado con las actitudes y conocimientos sobre abuso sexual (SAAKQ) (Bowman et al., 2010) y una escala de percepciones globales (GPS) (Scotti et al., 1996) sobre las personas con discapacidad. Los resultados indican que el taller es efectivo en cuanto a la adquisición de conocimientos sobre el abuso sexual, ya que se detecta un incremento significativo en las puntuaciones del SAAKQ en la evaluación post taller $(t(70)=9,4 ; p<0,001)$. Ahora bien, no obtienen diferencias estadísticamente significativas entre las puntuaciones previas y posteriores al taller en la percepción global.

\subsubsection{Planes de estudios}

El estudio restante basado en la educación sexual (Lund y Hammond, 2014) se dirige a la población susceptible de abuso. Lund y Hammond (2014) proponen un plan de estudios de educación de una sola sesión dirigido a personas adultas con discapacidad intelectual y del desarrollo que viven en áreas rurales. El plan consta de 4 módulos en los que se explican distintos tipos de abuso: abuso financiero, negligencia, abuso físico o verbal y abuso sexual. Este programa se evalúa mediante unas encuestas antes y después de impartir los 4 módulos de los que consta el plan. A pesar de que las autoras añaden información cualitativa de los resultados de estas encuestas y aseguran que el programa fue efectivo y las respuestas de las encuestas positivas, no se puede dar información fiable sobre los resultados ya que no se aportan datos numéricos que apoyen estas afirmaciones. 


\begin{tabular}{|c|c|c|c|c|c|c|}
\hline Referencia & Tipo de programa & Muestra & Entrenamiento & Métodos de evaluación & Resultados & Seguimiento \\
\hline $\begin{array}{l}\text { Bowman et al. } \\
\text { (2010) }\end{array}$ & $\begin{array}{l}\text { Educación sexual } \\
\text { Taller de prevención } \\
\text { Programa para } \\
\text { trabajadores con } \\
\text { personas con DID }\end{array}$ & $\begin{array}{l}n=124 \text { cuidadoras } \\
\text { jóvenes que trabajan } \\
\text { con personas con DID } \\
\text { Cl: s.d. } \\
\text { Edad: } M=30,1 \\
73,2 \% \text { mujeres } \\
26,8 \% \text { hombres }\end{array}$ & $\begin{array}{l}1 \text { sesión de } 4 \text { h, basada en: } \\
\text { - Defensa de abuso sexual } \\
\text { y leyes } \\
\text { - } \text { Factores de riesgo en } \\
\text { personas con DID } \\
\text { - Signos físicos y } \\
\text { conductuales } \\
\text { - Educación sexual } \\
\text { - Entornos seguros }\end{array}$ & $\begin{array}{l}\text { Pre y Post-intervención: } \\
\text { - SAAKQ } \\
\text { - GPS }\end{array}$ & $\begin{array}{l}\text { Comparación pre-post: } \\
\text { Puntuaciones SAAKQ } \\
\text { significativamente más altas en el } \\
\text { post entrenamiento en comparación } \\
\text { con el pre } \\
t(70)=9,4 \\
p<0,001 \\
\text { No cambios significativos en la } \\
\text { escala global (GPS) }\end{array}$ & No se hizo \\
\hline $\begin{array}{l}\text { Dryden et al. } \\
(2014,2016)\end{array}$ & $\begin{array}{l}\text { Adquisición } \\
\text { de habilidades } \\
\text { conductuales } \\
\text { Programa "IMPACT: } \\
\text { ability" } \\
\text { Sesiones de } \\
\text { capacitación } \\
\text { en seguridad y } \\
\text { autodefensa para } \\
\text { personas con DID }\end{array}$ & $\begin{array}{l}n=57 \text { estudiantes de } \\
\text { secundaria de escuelas } \\
\text { de educación especial } \\
\text { con DID } \\
\text { Gi: } n=21 \\
\text { Cl: s.d. } \\
\text { Edad: } M=17,4 \\
52,4 \% \text { mujeres } \\
47,6 \% \text { hombres } \\
\text { Gc: } n=36 \\
\text { Cl: } \text { s.d. } \\
\text { Edad: } \text { s.d } \\
55,6 \% \text { mujeres } \\
44,4 \% \text { hombres }\end{array}$ & $\begin{array}{l}10 \text { sesiones de } 90 \text { min cada } \\
\text { una, semanales, basadas en: } \\
\text { - } \quad \text { Establecer límites } \\
\text { - } \text { Informar y practicar } \\
\text { situaciones críticas } \\
\text { - Situaciones con sobornos } \\
\text { y amenazas } \\
\text { - Autodefensa }\end{array}$ & $\begin{array}{l}\text { Pre y Post-intervención: } \\
32 \text { preguntas sobre (Dryden } \\
\text { et al. 2014): } \\
\text { - Conocimiento seguridad } \\
\text { y autodefensa } \\
\text { - Confianza en defensa } \\
\text { personal } \\
\text { - Sentido de seguridad } \\
\text { - Seguridad al decir "no" } \\
\text { - Autodeterminación } \\
\text { - Autodefensa } \\
\text { - Autoeficacia }\end{array}$ & $\begin{array}{l}\text { Comparación entre grupos en el } \\
\text { post: } \\
\text { Puntuaciones significativamente } \\
\text { mejores en el grupo de intervención } \\
\text { en comparación con el grupo } \\
\text { control: } \\
\text { - Conocimiento seguridad y } \\
\text { autodefensa: } \\
p<0,0001 \\
\text { - Confianza en defensa personal } \\
p=0,033 \\
\text { - Seguridad al decir "no" } \\
p=0,017 \\
\text { Comparación pre-post: } \\
\text { Diferencias significativas pre/post en } \\
\text { el grupo de intervención, pero no en } \\
\text { el control: } \\
\text { - Conocimiento seguridad y } \\
\text { autodefensa: } \\
p<0,0001 \\
\text { - Confianza en defensa personal } \\
p=0,075 \\
\text { - Seguridad al decir "no" } \\
p=0,04 \\
\text { - Sentido de seguridad } \\
p=0,0066 \\
\text { - Autoeficacia } \\
p=0,046\end{array}$ & $\begin{array}{l}\text { A los } 12 \text { meses (Dryden et } \\
\text { al., 2016): (en el grupo de } \\
\text { intervención) } \\
\text { Se analizan las diferencias } \\
\text { entre los resultados } \\
\text { obtenidos en la encuesta } \\
2 \text { (la del seguimiento) con } \\
\text { respecto a la línea base } \\
\text { (Dif. Base, en adelante) y } \\
\text { la encuesta 1(Dif. E1, en } \\
\text { adelante) } \\
\text { Se obtienen diferencias } \\
\text { estadísticamente } \\
\text { significativas en: } \\
\text { - Conocimiento } \\
\text { seguridad y } \\
\text { autodefensa: } \\
\text {-Dif. Base: } p<0,01 \\
\text {-Dif. E.1: } p=0,0037 \\
\text { Autodefensa: } \\
\text {-Dif. Base: } p=0,01 \\
\text {-Dif. E.1: } p=0,04\end{array}$ \\
\hline $\begin{array}{l}\text { Egemo-Helm et } \\
\text { al. (2007) }\end{array}$ & $\begin{array}{l}\text { Adquisición } \\
\text { de habilidades } \\
\text { conductuales } \\
\\
\text { Programa de } \\
\text { entrenamiento (BST) } \\
\text { combinado con el } \\
\text { entrenamiento in } \\
\text { situ para enseñar } \\
\text { habilidades de } \\
\text { prevención del abuso } \\
\text { sexual }\end{array}$ & $\begin{array}{l}n=4 \text { mujeres con DID } \\
\text { leve-moderada } \\
\text { Cl: } M(3)=55,6 \\
\text { De una participante no } \\
\text { hay registro } \\
\text { Edad } 28-47 \text { años } \\
100 \% \text { mujeres }\end{array}$ & \begin{tabular}{|l|}
3 sesiones de 1 hora cada \\
una, de frecuencia sin \\
especificar, basadas en \\
habilidades conductuales: \\
- NO \\
- VAYA \\
- DIGA \\
Mediante: \\
- Juegos de rol \\
- Escenarios de situaciones \\
de abuso sexual
\end{tabular} & $\begin{array}{l}\text { Post-intervención: } \\
\text { - } \text { Autoinformes sobre cómo } \\
\text { actuaría en determinadas } \\
\text { situaciones. } \\
\text { - Juegos de rol } \\
\text { - Evaluación in situ } \\
\text { Situaciones basadas en } \\
\text { Lumley et al. } 1998\end{array}$ & $\begin{array}{l}\text { Tras el entrenamiento: } \\
\text { - } \quad \text { Autoinforme: } 4 \text { participantes } \\
\text { máxima puntuación (4 puntos) } \\
\text { - Juegos de rol: } 4 \text { participantes } \\
\text { máxima puntuación (4 puntos) } \\
\text { - Evaluación in situ: } 3 \text { participantes } \\
\text { máxima puntuación (4 puntos), } 1 \\
\text { participante } 2 \text { puntos } \\
\text { (Punt. máx. 4) }\end{array}$ & $\begin{array}{l}\text { A los } 3 \text { meses: } \\
2 \text { participantes obtuvieron } \\
\text { la máxima puntuación en } \\
\text { la evaluación y las otras } 2 \\
\text { obtuvieron puntuaciones } \\
\text { buenas (2 y } 3 \text { puntos) } \\
\text { (Punt. máx. 4) }\end{array}$ \\
\hline
\end{tabular}




\begin{tabular}{|c|c|c|c|c|c|c|}
\hline Referencia & Tipo de programa & Muestra & Entrenamiento & Métodos de evaluación & Resultados & Seguimiento \\
\hline Graff et al. (2018) & $\begin{array}{l}\text { Adquisición } \\
\text { de habilidades } \\
\text { conductuales } \\
\text { Programa "Opciones } \\
\text { Positivas" } \\
\text { para enseñar } \\
\text { habilidades sobre } \\
\text { sexualidad y } \\
\text { relaciones saludables }\end{array}$ & $\begin{array}{l}n=53 \text { estudiantes con } \\
\text { DID } \\
\text { Gi: (3 grupos) } \\
n=41 \\
\text { Cl: s.d. } \\
\text { Edad: } M=20,2 \\
48 \% \text { mujeres } \\
51 \% \text { hombres } \\
\text { Gc: } n=12 \\
\text { Cl: } \text { s.d. } \\
\text { Edad: } M=22,1 \\
66,6 \% \text { mujeres } \\
33,3 \% \text { hombres }\end{array}$ & $\begin{array}{l}5 \text { sesiones de duración y } \\
\text { frecuencia sin especificar } \\
\text { - Unidad 1: relaciones y } \\
\text { autoconciencia } \\
\text { - Unidad 2: maduración } \\
\text { - Unidad 3: ciclo de vida } \\
\text { (no se hace) } \\
\text { - Unidad 4: salud sexual } \\
\text { - Unidad 5: ser fuerte, } \\
\text { mantenerse a salvo }\end{array}$ & $\begin{array}{l}\text { Pre y Post-intervención: } \\
\text { - Actividades de toma de } \\
\text { decisiones } \\
\text { - Juegos de rol }\end{array}$ & $\begin{array}{l}\text { Comparación pre/post: Diferencias } \\
\text { significativas pre/post en el grupo } \\
\text { experimental, pero no en el control, } \\
\text { aunque sin especificar en cuál de los } \\
\text { métodos de evaluación: } \\
\text { - Unidad 1: } \\
\text { Gi: } p=0,000 \\
\text { Gc: } p=0,632 \\
\text { - Unidad 2: } \\
\text { Gi: } p=0,000 \\
\text { Gc: } p=1 \\
\text { - Unidad } 4 \\
\text { Gi: } p=0,002 \\
\text { Gc: } p=0,339 \\
\text { - Unidad } 5 \\
\text { Gi: } p=0,007 \\
\text { Gc: } p=0,185\end{array}$ & No se hizo \\
\hline $\begin{array}{l}\text { Hickson et al. } \\
\text { (2015) }\end{array}$ & $\begin{array}{l}\text { Adquisición } \\
\text { de habilidades } \\
\text { conductuales } \\
\text { Programa ESCAPE } \\
\text { DD. } \\
\text { Evaluar la capacidad } \\
\text { de toma de } \\
\text { decisiones en } \\
\text { personas con DID en } \\
\text { situaciones de abuso } \\
\text { sexual }\end{array}$ & $\begin{array}{l}n=58 \text { personas con } \\
\text { DID leve-moderada } \\
\text { CI: } M=56,78 \\
\text { Gi: } n=30 \\
\text { Edad: } M=39,4 \\
46 \% \text { mujeres } \\
54 \% \text { hombres } \\
\text { Gc: } n=28 \\
\text { Edad: } M=38,2 \\
53,5 \% \text { mujeres } \\
46,5 \% \text { hombres }\end{array}$ & $\begin{array}{l}12 \text { sesiones de } 1 \mathrm{~h} \text { aprox. } \\
\text { Cada semana o cada dos } \\
\text { semanas, basadas en: } \\
\text { - Unidad 1: conceptos } \\
\text { básicos y objetivos de } \\
\text { autoprotección } \\
\text { - Unidad 2: modelar } \\
\text { toma de decisiones y } \\
\text { proporcionar práctica } \\
\text { Mediante: viñetas que } \\
\text { representan situaciones de } \\
\text { abuso sexual }\end{array}$ & $\begin{array}{l}\text { Pre y Post-intervención: } \\
\text { Escala de toma de } \\
\text { decisiones (DMS) basada } \\
\text { en viñetas que reflejan } \\
\text { situaciones de abuso } \\
\text { sexual: ¿qué está pasando? } \\
\text { y ¿qué debería hacer el } \\
\text { protagonista? }\end{array}$ & $\begin{array}{l}\text { Comparación entre grupos en el } \\
\text { post: } \\
\text { Puntuaciones significativamente más } \\
\text { altas en el grupo de intervención en } \\
\text { comparación con el grupo control: } \\
\text { - Toma de decisiones efectiva en } \\
\text { general } \\
F(1,52)=6,61 \\
p=0,013 \\
\eta 2=0,113 \\
\text { - Toma de decisiones efectiva y } \\
\text { segura ahora } \\
F(1,52)=5,73 \\
p=0,02 \\
\eta 2=0,099 \\
\text { Comparación pre-post: } \\
\text { Diferencias significativas pre/post en } \\
\text { el grupo experimental, pero no en el } \\
\text { control: } \\
\text { - Toma de decisiones efectiva en } \\
\text { general } \\
\text { Gi: } t(29)=4,27 \\
p=0,001 \\
\text { Toma de decisiones efectiva y } \\
\text { segura ahora } \\
\text { Gi: } t(29)=2,56 \\
p=0,05\end{array}$ & No se hizo \\
\hline
\end{tabular}




\begin{tabular}{|c|c|c|c|c|c|c|}
\hline Referencia & Tipo de programa & Muestra & Entrenamiento & Métodos de evaluación & Resultados & Seguimiento \\
\hline Kim (2016) & $\begin{array}{l}\text { Adquisición } \\
\text { de habilidades } \\
\text { conductuales } \\
\\
\text { Programa de } \\
\text { prevención del abuso } \\
\text { sexual en niñas con } \\
\text { DID }\end{array}$ & $\begin{array}{l}n=3 \text { adolescentes } \\
\text { coreanas con DID leve- } \\
\text { moderado } \\
\text { Cl: } M=56,33 \\
\text { Edad: } M=12,5 \\
100 \% \text { mujeres }\end{array}$ & $\begin{array}{l}5 \text { sesiones de } 30 \text { min cada } \\
\text { una, } 2 \text { o } 3 \text { veces por semana, } \\
\text { basadas en habilidades } \\
\text { conductuales: } \\
\text { - } \text { NO } \\
\text { - VAYA } \\
\text { - DIGA } \\
\text { Mediante: } \\
\text { - Posters (partes del } \\
\text { cuerpo). } \\
\text { - Juegos de tarjetas } \\
\text { - } \text { (apropiado/no apropiado) } \\
\text { represento para colorear que } \\
\text { de abuso sexual resueltas } \\
\text { correctamente (cómo } \\
\text { rechazar) } \\
\text { - Juegos de rol } \\
\text { - Escenarios } \\
\end{array}$ & $\begin{array}{l}\text { Pre y Post-intervención: } \\
\text { - Juegos de rol } \\
\text { - Evaluación in situ }\end{array}$ & $\begin{array}{l}\text { Comparación pre-post sin } \\
\text { especificar en qué método de } \\
\text { evaluación: } \\
\text { - Participante 1: } \\
\text { Pre: } 0 \\
\text { Post: } 4 \\
\text { Generalización: } 4 \\
\text { - Participante 2: } \\
\text { Pre: } 0 \\
\text { Post: } 4 \\
\text { Generalización: } 4 \\
\text { - Participante } 3: \\
\text { Pre: } 1 \\
\text { Post: } 4 \\
\text { Generalización: } 3 \\
\text { (Punt. máx. 4) }\end{array}$ & $\begin{array}{l}\text { A las } 10 \text { semanas: } \\
\text { - } \quad \text { Participante } 1: 4 \\
\text { - } \quad \text { Participante 2: } 4 \\
\text { (Punt. máx. 4) }\end{array}$ \\
\hline $\begin{array}{l}\text { Lumley et al. } \\
\text { (1998) }\end{array}$ & $\begin{array}{l}\text { Adquisición } \\
\text { de habilidades } \\
\text { conductuales } \\
\\
\text { Programa de } \\
\text { prevención del abuso } \\
\text { sexual en personas } \\
\text { con DID }\end{array}$ & $\begin{array}{l}n=6 \text { mujeres con DID } \\
\text { leve-moderada } \\
\text { Cl: s.d. } \\
\text { Edad: } 30-42 \text { años } \\
100 \% \text { mujeres }\end{array}$ & $\begin{array}{l}5 \text { sesiones de } 60 \text { a } 90 \text { min } \\
\text { cada una, basadas en } \\
\text { habilidades conductuales: } \\
\text { - } \quad \text { NO (rechazar) } \\
\text { - } \text { VAYA (abandonar) } \\
\text { - DIGA (informar) } \\
\text { Mediante: } \\
\text { - Juegos de rol } \\
\text { - Escenarios de situaciones } \\
\quad \text { de abuso sexual }\end{array}$ & $\begin{array}{l}\text { Pre y Post-intervención: } \\
\text { - Informe verbal sobre el } \\
\text { conocimiento de abuso } \\
\text { sexual } \\
\text { - Juegos de rol } \\
\text { - Evaluación in situ }\end{array}$ & $\begin{array}{l}\text { Comparación pre-post*: } \\
\text { - Informe verbal } \\
\text { Pre: } M=1,6 \\
\text { Post: } M=3,6 \\
\text { - Juegos de rol } \\
\text { Pre: } M=1,3 \\
\text { Post: } M=4 \\
\text { - In situ } \\
\text { Pre: } M=1,3 \\
\text { Post: } M=1,8 \\
\text { (Punt. máx. 4) }\end{array}$ & \begin{tabular}{|l} 
Al mes: \\
- \\
- Informe verbal $M=2,5$ \\
Juegos de rol \\
$M=3,6$ \\
- In situ \\
$M=1,3$ \\
(Punt. máx. 4)
\end{tabular} \\
\hline $\begin{array}{l}\text { Lund y } \\
\text { Hammond } \\
\text { (2014) }\end{array}$ & $\begin{array}{l}\text { Educación sexual } \\
\text { Intervención para la } \\
\text { conciencia del abuso } \\
\text { sexual en personas } \\
\text { con DID }\end{array}$ & $\begin{array}{l}\text { Personas adultas con } \\
\text { DID que viven en zonas } \\
\text { rurales } \\
n=\text { s. d. } \\
\text { Cl: s.d. } \\
\text { Edad: s.d. } \\
\text { Porcentajes de } \\
\text { hombres y mujeres: s.d. }\end{array}$ & $\begin{array}{l}1 \text { sesión de duración } \\
\text { desconocida, basada en } 4 \\
\text { módulos: } \\
\text { - Abuso financiero } \\
\text { - Negligencia } \\
\text { - Abuso físico o verbal } \\
\text { - } \quad \text { Abuso sexual }\end{array}$ & $\begin{array}{l}\text { Pre y Post-intervención: } \\
\text { - } \quad \text { Conceptos clave del } \\
\text { abuso } \\
\text { - Seguridad en casa, } \\
\text { trabajo y comunidad }\end{array}$ & $\begin{array}{l}\text { - Satisfacción general se evaluó } \\
\text { en una encuesta y fue alta: } \\
\text { experiencia general del taller fue } \\
\text { positiva. } \\
\text { - Comentarios de los facilitadores/ } \\
\text { as también indican que la } \\
\text { experiencia fue positiva. }\end{array}$ & No se hizo \\
\hline
\end{tabular}




\begin{tabular}{|c|c|c|c|c|c|c|}
\hline Referencia & Tipo de programa & Muestra & Entrenamiento & Métodos de evaluación & Resultados & Seguimiento \\
\hline $\begin{array}{l}\text { Miltenberger et } \\
\text { al. (1999) }\end{array}$ & $\begin{array}{l}\text { Adquisición } \\
\text { de habilidades } \\
\text { conductuales } \\
\\
\text { Programa de } \\
\text { prevención del abuso } \\
\text { sexual en personas } \\
\text { con DID }\end{array}$ & $\begin{array}{l}n=5 \text { mujeres con DID } \\
\text { leve-moderada } \\
\text { Cl: s.d. } \\
\text { Edad: } 33-57 \text { años } \\
100 \% \text { mujeres }\end{array}$ & \begin{tabular}{|l}
10 sesiones de 1 hora cada \\
una, semanales, basadas en: \\
- Información sobre abuso \\
- sexual \\
- Habilidades de prevención \\
- Ensayo de habilidades \\
Mediante: \\
- Juegos de rol \\
- Escenarios y situaciones \\
de abuso sexual
\end{tabular} & $\begin{array}{l}\text { Pre y Post-intervención: } \\
\text { - Informes verbales } \\
\text { - Juegos de rol } \\
\text { - } \quad \text { Evaluación in situ }\end{array}$ & $\begin{array}{l}\text { Comparación pre/post*: } \\
\text { - Informe verbal } \\
\text { Pre: } M=1,4 \\
\text { Post: } M=2,4 \\
\text { - Juego de rol } \\
\text { Pre: } M=1,4 \\
\text { Post: } M=4 \\
\text { - In situ } \\
\text { Pre: } M=0,6 \\
\text { Post: } M=3,6 \\
\text { (Punt. máx. 4) }\end{array}$ & $\begin{array}{l}\text { Al mes: } \\
\text { - Juego de rol } \\
M=4 \\
\text { - In situ } \\
\quad M=3 \\
\text { (Punt. máx. 4) }\end{array}$ \\
\hline
\end{tabular}

*En los artículos no se aportan los datos correspondientes a las desviaciones típicas que acompañan las medias

Nota: s.d.: sin datos; Cl: cociente intelectual; DID: discapacidad intelectual y del desarrollo; SAAKQ: Sexual Abuse Attitudes and Knowledge Questionnaire (Bowman et al., 2010); GPS: Global Perceptions Scale (Scotti et al., 1996); IMPACT: ability: program (Dryden et al., 2014); Gi: Grupo de intervención; Gc: Grupo control; BST: behavioral skills training (Haseltine y Miltenberger, 1990); ESCAPE-DD: An effective strategybased curriculum for abuse prevention and empowerment for adults with developmental disabilities (Khemka y Hickson, 2008); DMS: Decision-Making Scale (Hickson et al., 2015).

Fuente: elaboración propia. 


\section{Discusión}

En este estudio se ha revisado sistemáticamente la literatura científica publicada hasta la fecha sobre la prevención del abuso sexual en personas con discapacidad intelectual y del desarrollo. Concretamente, se han revisado 10 artículos sobre el tema. El número de artículos revisados lleva a plantear una primera conclusión y es que existen muy pocos estudios empíricos en este campo, una carencia que se hace especialmente significativa teniendo en cuenta que, tal y como señalan algunos autores y autoras (por ejemplo, Couwenhoven, 2013), más de la mitad de las personas que tienen discapacidad intelectual y del desarrollo son vulnerables a sufrir abuso sexual, con las importantes secuelas psicológicas que este tipo de situaciones pueden llegar a suponer.

La escasez de investigación en este campo es todavía más dramática si se tiene en cuenta que en el caso de las mujeres con discapacidad intelectual y del desarrollo, el porcentaje de abuso sexual es todavía mayor (Couwenhoven, 2013; Martinello, 2014). Sin embargo, la ya mencionada discriminación interseccional que sufren las mujeres con discapacidad intelectual y del desarrollo en cuanto al abuso sexual (Cavalcante, 2018; CERMI, 2013; Gomiz, 2016; Viñuela, 2009), concuerda con el hecho de que casi todos los estudios analizados en esta revisión se han realizado con una muestra mayoritaria o exclusiva de mujeres, con un único estudio sobre el que no se ha podido averiguar si esto era así, dada la falta de datos descriptivos sobre la muestra empleada (Lund y Hammond, 2014). Por lo tanto, la segunda conclusión que puede extraerse de esta revisión sistemática es que los estudios examinados, aunque escasos, son de alguna manera representativos de la realidad que nos ocupa y que, por tanto, sus resultados deberán tener una lectura femenina, puesto que todas las intervenciones se han puesto a prueba fundamentalmente, en mujeres.

Tal y como se mencionaba en el apartado anterior, los estudios sobre este tema se dividieron en dos grupos: programas centrados en la adquisición de habilidades conductuales (Dryden et al., 2014, 2016; EgemoHelm et al., 2007; Graff et al., 2018; Hickson et al., 2015; Kim, 2016; Lumley et al., 1998; Miltenberger et al., 1999) y programas de educación sexual (Bowman et al., 2010; Lund y Hammond, 2014).

En el primer grupo, la mayoría de los estudios están basados en el entrenamiento de habilidades en escenarios ficticios sobre situaciones apropiadas e inapropiadas, con el fin de que los y las participantes aprendan lo que es y lo que no es adecuado que les suceda. Además, estos programas se centran en dotar de herramientas para identificar y defenderse ante un abuso. Los programas de este primer tipo son más completos que los del segundo, debido a que todos ellos incluyen, en mayor o menor medida, psicoeducación sobre abuso sexual antes de enseñar habilidades conductuales al respecto.

Estos programas para la adquisición de habilidades conductuales muestran en general resultados positivos, aunque entre ellos hay distintos grados de robustez en cuanto a la metodología utilizada. De los 8 programas que enseñan habilidades conductuales, el subgrupo de 3 estudios que utilizan un entrenamiento basado en role-playing combinado con otros tipos de entrenamiento (Dryden et al., 2014; Graff et al., 2018; Hickson et al., 2015) tienen un mejor diseño, pues disponen de un grupo de comparación o grupo control. El estudio de Hickson et al. (2015), además, sigue un diseño experimental, ya que el procedimiento de asignación al grupo de intervención o grupo de control de lista de espera es al azar, mientras que el programa de Graff et al. (2018) y el estudio de Dryden et al. (2014) siguen un diseño cuasi-experimental. Sea como fuere, en los tres casos se encuentra que el grupo de intervención tiene mejores resultados que el grupo control, 
con diferencias significativas pre-post en el grupo de intervención, pero no así en el grupo control $(p \leq 0,05)$. Además, Dryden et al. (2014) y Hickson et al. (2015) muestran análisis de comparación entre grupos, con datos a favor del grupo de intervención en todas las escalas medidas $(p \leq 0,05)$. Incluso en el estudio de Dryden et al. (2016) se hace un seguimiento tras 12 meses, donde se observan resultados positivos en el grupo de intervención.

A pesar de su mayor rigor metodológico y de sus buenos resultados, estos estudios no parecen ser demasiado exigentes a la hora de medir sus resultados, pues se basan únicamente en escalas de toma de decisiones y cuestionarios, sin medir realmente las habilidades adquiridas por los y las participantes en situaciones reales.

El grupo de estudios basado en el protocolo de Lumley et al. (1998) (Egemo-Helm et al., 2007; Kim, 2016; Lumley et al., 1998; Miltenberger et al., 1999), utilizan este protocolo más práctico, caracterizado por la adquisición de habilidades para rechazar, abandonar e informar de la situación de la que se está siendo víctima, y de manera coherente, evalúan los resultados de dicho protocolo a partir de juegos de rol y evaluación in situ. En estos estudios, se encuentran resultados positivos que además se mantienen a largo plazo, pero sus muestras son mucho más pequeñas y probablemente como consecuencia, los resultados son mucho más endebles estadísticamente hablando, ya que no muestran más que puntuaciones directas de cada participante (Egemo-Helm et al., 2007; Kim, 2016) o medias de antes y después del entrenamiento (Lumley et al., 1998; Miltenberger et al., 1999), sin utilizar en ningún caso estadísticos de comparación entre las medidas pre y post intervención. Por ello, aunque los resultados parecen ser positivos, en realidad se carece de herramientas para juzgar si los programas de entrenamiento a los que se refieren son efectivos realmente o no. Además, el tamaño muestral tan pequeño impediría la generalización de los resultados a la población general de personas con discapacidad intelectual y del desarrollo. Por último, a pesar de que este grupo de estudios es el único que muestra de manera general resultados de seguimiento para observar si la adquisición de las habilidades se mantiene en el tiempo, e incluso, parece que estos resultados son positivos, solamente muestran datos descriptivos, por lo que una vez más, es imposible sacar conclusiones contundentes de los mismos.

Por otro lado, los estudios sobre programas de educación sexual (Bowman et al., 2010; Lund y Hammond, 2014) muestran todavía menos resultados sobre la efectividad de las intervenciones. Solo uno de ellos (Bowman et al., 2010) aporta datos estadísticos sobre los resultados del tratamiento, con diferencias pre y post significativas $(p<0,001)$, aunque no dispone de un grupo control de comparación y, además, la muestra sigue siendo pequeña y con ello, poco representativa. En el caso de Lund y Hammond (2014), solo mencionan la efectividad del entrenamiento haciendo referencia a que las respuestas recibidas de las encuestas fueron positivas, pero no aportan ningún dato cuantitativo sobre ello.

Por lo tanto, la tercera conclusión que podemos sacar de esta revisión es que, a día de hoy y hasta que no se haga más investigación y de mayor calidad en este campo, son los programas de habilidades, al menos del tipo de los dos únicos estudios que muestran comparaciones entre grupos (Hickson et al., 2015; Dryden et al., 2014), los que constituyen la primera línea de actuación para prevenir el abuso sexual en la población con discapacidad intelectual y del desarrollo de manera general, y en la población de mujeres con discapacidad intelectual y del desarrollo de manera particular, puesto que, considerando las muestras de ambos estudios en su conjunto, las mujeres constituyen la mayoría de participantes. No obstante, es importante recordar que se trata solamente de dos estudios, que uno de ellos sigue un diseño cuasi-experimental y que ninguno incluye medidas in situ. Asimismo, también parece conveniente subrayar que, a pesar de ser 
programas ofertados básicamente a mujeres con discapacidad intelectual y del desarrollo, el objetivo de los protocolos de tratamiento es paliar las causas de la vulnerabilidad asociada a la discapacidad intelectual y del desarrollo en general y no en el caso de las mujeres con discapacidad intelectual y del desarrollo en particular. Esto pondría de relieve, tal y como señalaban en su revisión Barger et al. (2009), la inexistencia de recursos de prevención específicos para las mujeres con discapacidad intelectual y del desarrollo.

En cualquier caso, los resultados obtenidos tras la presente revisión coinciden, en parte, con los resultados de las revisiones previas de este ámbito (Barger et al., 2009; Doughty y Kane, 2010; Mikton et al., 2014). Barger et al. (2009) concluyen que los programas que revisaron tenían deficiencias, ya que la efectividad de estos se medía solo por la satisfacción de los y las participantes, menos uno de ellos que aportaba datos numéricos sobre la efectividad del programa. Doughty y Kane (2010) centran su revisión en la generalización de las habilidades adquiridas durante el entrenamiento y concluyen que, en cierto modo, estas habilidades se mantuvieron y generalizaron, pero tampoco aportan datos estadísticos al respecto. Por último, Mikton et al. (2014) afirman que de los 10 programas que evalúan siete fueron poco claros y tres ineficaces, tras calificar los estudios como débiles en QATQS (herramienta de evaluación de calidad para estudios cuantitativos).

Los resultados obtenidos en esta revisión sobre la efectividad de los programas de prevención del abuso sexual en personas con discapacidad intelectual y del desarrollo también coinciden con los resultados obtenidos en una revisión sobre programas de prevención del abuso sexual en niños y niñas, que son también considerados población vulnerable. Del Campo y Fávero (2020) revisan programas que se clasifican en cuatro grupos: aumento de conocimiento sobre abuso sexual, adquisición de habilidades de afrontamiento, cambios que tienen lugar en la vida real y posibles efectos negativos. Este trabajo concluye que la mayoría de los estudios analizados miden la efectividad de los programas mediante cuestionarios que evalúan el conocimiento antes y después del entrenamiento y mediante juegos de rol y evaluación in situ que evalúan las habilidades de protección. También afirman que hay un aumento significativo en las medidas después del entrenamiento, y en los casos en los que se compara un grupo control con un grupo de intervención, este último obtiene mejores puntuaciones, pero tampoco aportan datos numéricos al respecto.

Pero esta falta de estudios no parece estar relacionada únicamente con el ámbito del abuso sexual, sino que parece un mal endémico de los estudios aplicados a la población con discapacidad intelectual y del desarrollo. Así, la falta de estudios sobre abuso sexual en personas con discapacidad intelectual y del desarrollo, es coherente también con la falta de estudios que existe respecto a esta población en otros ámbitos, como, por ejemplo, en el ámbito forense. De hecho, Fisher et al. (2015), realizan una revisión sobre la victimización y la vulnerabilidad social de personas con discapacidad intelectual y del desarrollo en la que concluyen que existe una escasez de programas de prevención en esta área.

Asimismo, aunque sabemos que el abuso sexual puede generar importantes consecuencias psicológicas en las personas con discapacidad intelectual y del desarrollo, entre ellas, por ejemplo, TEPT (Brackenridge y Morrissey, 2010; Recio et al., 2013), también se puede observar una escasez de estudios de calidad a este respecto. Mevissen y de Jongh (2010) en su revisión de programas de intervención para personas con discapacidad intelectual y del desarrollo que sufren TEPT concluyen que faltan instrumentos de diagnóstico específico para esta población y que, por tanto, no se pueden establecer tasas de prevalencia del trastorno fiables en este campo. De la misma manera, exponen que tampoco existen métodos de tratamiento empíricamente validados para el TEPT en personas con discapacidad intelectual y del desarrollo. 
En general, todos estos datos nos demuestran que, aunque la falta de estudios sobre abuso sexual en personas con discapacidad intelectual y del desarrollo, sobre todo en mujeres, es muy llamativa, faltan estudios relacionados con la generalidad de la población con discapacidad intelectual y del desarrollo, que es una población olvidada y que está rodeada de muchos mitos que interfieren en su calidad de vida y en su desarrollo personal.

Aunque las conclusiones que se pueden extraer de esta revisión son útiles, por supuesto, este trabajo no está exento de limitaciones. En relación con el propio procedimiento de revisión sistemática, el hecho de que las dos investigadoras que han realizado este trabajo no hayan sido completamente independientes, puede suponer un sesgo dentro de la metodología de revisión sistemática (Liberati et al., 2009). Además, el uso de una síntesis meramente cualitativa y no cuantitativa de los resultados, hace que la información que puede extraerse de esta revisión sistemática no sea tan completa como lo sería si se incluyesen los métodos estadísticos que se suelen utilizar en los metaanálisis para combinar los resultados de diferentes estudios, evaluar su heterogeneidad, estimar la influencia de distintos factores o covariables y evaluar la presencia de sesgos de publicación, todo lo cual supone una de las principales aportaciones del protocolo PRISMA (Moher et al., 2009). No obstante, es importante recordar que todos estos procedimientos también necesitan de un número mayor de estudios individuales para su correcta aplicación, por lo que habrá que esperar a que la literatura científica sobre este tema sea más amplia.

También existen limitaciones relacionadas con los estudios revisados. Como consecuencia obvia de la conclusión anterior, la limitación más importante con la que cuenta este estudio de revisión es el haber dispuesto de pocas investigaciones que analizar y de pocos datos en los que sustentarse, por lo que no se han podido sacar conclusiones demasiado decisivas, más allá de que este campo necesita mucha investigación, más específica y de mejor calidad. Otra limitación de este estudio es que la revisión incluye únicamente trabajos centrados en personas con discapacidad intelectual y del desarrollo, sin incluir otros tipos de discapacidades, como la ya mencionada discapacidad psicosocial (Drew et al., 2011) o incluso, la discapacidad de aprendizaje, la cual comparte algunos aspectos con la discapacidad intelectual y del desarrollo, entre ellos, la vulnerabilidad de estas personas a sufrir abuso y los problemas de comunicación y lenguaje (Griveo et al., 2006). Asimismo, la mayoría de los programas de prevención analizados están dirigidos a personas con discapacidad intelectual y del desarrollo leve-moderada, olvidándose de las personas con discapacidad intelectual y del desarrollo grave, que son también vulnerables a sufrir abuso.

Como ya se ha mencionado, la metodología de los estudios analizados también supone una gran limitación para los propósitos de esta revisión. Los tamaños muestrales de los estudios, en general, son pequeños. Además, aunque el hecho de que haya más mujeres parece ser más representativo del fenómeno, algunos estudios han señalado este aspecto como una posible fuente de sesgo en la interpretación de los resultados obtenidos (Doughty y Kane, 2010). Por otro lado, los instrumentos de medida en cada programa son muy variados y diferentes, lo que no permite establecer comparaciones justas de los resultados obtenidos tras las mediciones ni emplear estadísticos que permitan combinar los resultados en forma de síntesis cuantitativa (metaanálisis). Otra limitación es que la mayoría de los estudios son transversales y muy pocos hacen seguimientos y cuando los hacen, no se aportan más que datos descriptivos.

Ante los resultados y las limitaciones observados tras la revisión se destaca que esta es una importante área para futuras investigaciones, puesto que está casi todo por hacer. La investigación futura debería ocuparse de las personas con discapacidad intelectual y del desarrollo grave, se deben realizar estudios empíricos con muestras más grandes, mejores diseños y análisis estadísticos que permitan extraer conclusiones de 
datos numéricos y fiables. Por último, deberían hacerse estudios no solo transversales, sino longitudinales tal y como refieren también Barger et al. (2009), y tanto de habilidades conductuales como de educación sexual. Adicionalmente, los programas de prevención del abuso sexual seguramente deberían adoptar una perspectiva de género más clara, adaptando sus objetivos y técnicas a la interseccionalidad de las mujeres con discapacidad intelectual y del desarrollo.

En cualquier caso, la revisión llevada a cabo en este trabajo muestra que, aunque parece que los programas de prevención, especialmente de habilidades conductuales, podrían ser efectivos para paliar el problema de los abusos sexuales en personas con discapacidad intelectual y del desarrollo, fundamentalmente mujeres, se necesita mucha más investigación en este ámbito para poder asegurar esto. En una población tan vulnerable y que sufre en gran medida las consecuencias psicológicas de los abusos, realizar más investigación es prioritario. 
Alemany, A. et al. (2012). Guía de intervención policial con personas con discapacidad intelectual. Fundación Carmen Pardo-Valcarce.

American Psychiatric Association-APA (2014). Guía de consulta de criterios diagnósticos del DSM-5: Spanish edition of the desk Reference to the diagnostic criteria from DSM-5. American Psychiatric Pub.

Barger, E. et al. (2009). Sexual assault prevention for women with intellectual disabilities: a critical review of the evidence. Intellectual and Developmental Disabilities, 47(4), pp. 249-262. https://doi.org/10.1352/1934-955647.4.249.

Bowman, R. A. et al. (2010). Sexual abuse prevention: a training program for developmental disabilities service providers. Journal of Child Sexual Abuse, 19, pp.119-127. https://doi.org/10.1080/10538711003614718.

Brackenridge, I. y Morrissey, C. (2010). Trauma and post-traumatic stress disorder (PTSD) in high secure forensic learning disability population: future direction for practice. Advances in Mental Health Intellectual Disabilities, 4, pp. 49-56. https://doi.org/10.5042/amhid.2010.0544.

Brown, H. y Turk, V. (1992). Defining sexual abuse as it affects adults with learning disabilities. Mental Handicap, 20, pp. 33-55. https://doi.org/10.1111/j.1468-3156.1992.tb00658.x.

Bruder, C. y Kroese, B. S. (2005). The efficacy of interventions designed to prevent and protect people with intellectual disabilities from sexual abuse: a review of the literature. The Journal of Adult Protection, 7, pp. 13-27. https://doi.org/10.1108/14668203200500009.

del Campo, A. y Fávero, M. (2020). Effectiveness of Programs for the Prevention of Child Sexual Abuse". European Psychologist, 25(1), pp. 1-15. https://doi.org/10.1027/1016-9040/a000379.

Cavalcante, A. M. (2018). Discriminación interseccional: concepto y consecuencias en la incidencia de violencia sexual contra mujeres con discapacidad. Journal of Feminist, Gender and Women Studies, 7, pp. 15-25. http:// dx.doi.org/10.15366/jfgws2018.7.002.

Chodan, W. et al. (2017). A randomized controlled trial on the effectiveness of a sexual abuse prevention programme for girls with intellectual disabilities: study protocol. Translational Developmental Psychiatric, 5(1), pp. 1407192. https://doi.org/10.1080/20017022.2017.1407192.

Chrastina, J. y Vecerova, H. (2018). Supporting Sexuality in Adults with Intellectual Disability- A short Review. Sexuality and Disability, 38, pp. 285-298. https://doi.org/10.1007/s11195-018-9546-8.

Comité Español de Representantes de Personas con Discapacidad, CERMI (2013). La transversalidad de género en las políticas públicas sobre discapacidad. Volumen II. Cinca.

Couwenhoven, T. (2013). La educación sexual es la prevención del abuso sexual. Revista Síndrome de Down, 30 , pp. 9-14.

Doughty, H. A. y Kane, M. L. (2010). Teaching abuse-protection skills to people with intellectual disabilities: A review of the literature. Research in Developmental Disabilities, 31, pp. 331-337. https://doi.org/10.1016/j. ridd.2009.12.007.

Drew, N. et al. (2011). Human rights violations of people with mental and psychosocial disabilities: an unresolved global crisis. The Lancet, 378, pp. 1664-1675. https://doi.org/10.1016/S0140-6736(11)61458-X. 
Dryden, E. M. et al. (2014). Effectiveness of the IMPACT: Ability program to improve safety and self-advocacy skills in high school students with disabilities. Journal of School Health, 84(12), pp. 793-801. https://doi.org/10.1111/ josh.12211.

Dryden, E. M. et al. (2016). Effectiveness of IMPACT: ability to improve safety and self-advocacy skills in students with disabilities - follow-up study. Journal of School Health, 87, pp. 83-89. https://doi.org/10.1111/josh.12474.

Egemo-Helm, K. et al. (2007). An evaluation of in situ training to teach sexual abuse prevention skills to women with mental retardation. Behavioral Interventions, 22, pp. 99-119. https://doi.org/10.1002/bin.234.

España. Ley Orgánica 10/1995, de 23 de noviembre, del Código Penal. Boletín Oficial del Estado, 24 de noviembre de 1995, núm. 281, pp. 33987-34058.

Euser, S. et al. (2015). The prevalence of child sexual abuse in out-of-home care: increased risk for children with a mild intellectual disability. Journal of Applied Research in Intellectual Disabilities, 29, pp. 83-92. https://doi. org/10.1111/jar.12160.

Finkelhor, D. et al. (1990). Sexual abuse in a national survey of adult men and women: Prevalence, characteristics and risk factor. Child Abuse and Neglect, 14, pp. 19-28. https://doi.org/10.1016/0145-2134(90)90077-7.

Fisher, M. H. et al. (2015). Victimization and Social Vulnerability of Adults with Intellectual Disability: a review of research extending beyond Wilson and Brewer. Australian Psychologist, 51, pp. 114-127. https://doi.org/10.1111/ ap. 12180 .

Giménez-García, C. et al. (2017). Abuso sexual y discapacidad intelectual: cómo identifican y valoran la experiencia las personas con discapacidad intelectual y los profesionales que les atiendes. International Journal of Developmental and Educational Psychology, 4, pp. 129-136. https://doi.org/10.17060/ijodaep.2017.n1.v4.1035.

Gomiz, M. P. (2016). La sexualidad y la maternidad como factores adicionales de discriminación (y violencia) en las mujeres con discapacidad. Revista Española de Discapacidad, 4(2), pp. 123-142. https://doi.org/10.5569/23405104.04.02.07.

Graff, H. J. et al. (2018). Relationships and Sexuality: How is a Young Adult with Intellectual Disability Supossed to Navigate?. Sex Disabil, 36, pp. 175-183. https://doi.org/10.1007/s11195-017-9499-3.

Griveo, A. et al. (2006). An evaluation of research and training resources for the sex education of people with moderate to severe learning disabilities. British Journal of Learning Disabilities, 35, pp. 30-37. https://doi. org/10.1111/j.1468-3156.2006.00401.x.

Gutiérrez de Piñeres, C. et al. (2009). Revisión teórica del concepto de victimización secundaria. Liberabit, 15(1), pp. 49-58.

Haseltine, B. y Miltenberger, R. G. (1990). Teaching self-protection skills to persons with mental retardation. American Journal on Mental Retardation, 95, pp. 188-197.

Hickson, L. et al. (2015). Randomized controlled trial to evaluate an abuse prevention curriculum for women and men with intellectual and developmental disabilities. American Journal on Intellectual and Developmental Disabilities, 6, pp. 490-503. https://doi.org/10.1352/1944-7558-120.6.490.

Khemka, I. y Hickson, L. (2008). ESCAPE-DD: An effective strategy-based curriculum for abuse prevention and empowerment for adults with developmental disabilities. Center for Opportunities and Outcomes for People with Disabilities, Teachers College, Columbia University. 
Kim, Y. (2016). Evaluation of a sexual abuse prevention program for children with intelectual disabilities. Behavioral Interventions, 31, pp. 195-209. https://doi.org/10.1002/bin.1439.

Liberati, A. et al. (2009). The PRISMA statement for reporting systematic reviews and meta-analyses of studies that evaluate health care interventions: explanation and elaboration. PLoS Med 6(7), pp. e1000100. https://doi. org/10.1371/journal.pmed.1000100.

Lin, L. P. et al. (2009). Sexual assault of people with disabilities: results of a 2002-2007 national report in Taiwan. Research in Developmental Disabilities, 30, pp. 969-975. https://doi.org/10.1016/j.ridd.2009.02.001.

Lumley, V. A. et al. (1998). Evaluation of a sexual abuse prevention program for adults with mental retardation. Journal of Applied Behavior Analysis, 31, pp. 91-101. https://doi.org/10.1901/jaba.1998.31-91.

Lund, E. M. y Hammond, M. (2014). Single-session intervention for abuse awareness among people with developmental disabilities. Sex Disabil, 32, pp. 99-105. https://doi.org/10.1007/s11195-013-9335-3.

Martin S. L. et al. (2006). Physical and sexual assault of women with disabilities. Violence Against Women, 12, pp. 823-837. https://doi.org/10.1177/1077801206292672.

Martinello, E. (2014). Reviewing Strategies for Risk Reduction of Sexual Abuse of Children with Intellectual Disabilities: A Focus on Early Intervention. Sex Disabil, 32, pp. 167-174. https://doi.org/10.1007/s11195-014-9345-9.

McCarthy, M. (1993). Sexual experiences of women with learning disabilities in long stay hospitals. Sexuality and Disability, 11, pp. 277-286. https://doi-org.bucm.idm.oclc.org/10.1007/BF01102172.

Mevissen, L. y de Jongh, A. (2010). PTSD and its treatment in people with intellectual disabilities. A review of the literature. Clinical Psychology Review, 30, pp. 308-316. https://doi.org/10.1016/j.cpr.2009.12.005.

Mikton, C. et al. (2014). A systematic review of the effectiveness of intervention to prevent and respond to violence against personas with disabilities. Journal of Interpersonal Violence, pp. 1-20. https://doi. org/10.1177/0886260514534530.

Miltenberger, R. G. et al. (1999). Training and generalization of sexual abuse prevention skills for women with mental retardation. Journal of Applied Behavior Analysis, 32, pp. 385-388. https://doi.org/10.1901/jaba.1999.32-385.

Moher, D. et al. (2009). Preferred reporting items for systematic reviews and meta-analyses: the PRISMA statement. PLoS Medicine, 6(7), pp. e1000097. https://doi.org/10.1371/journal.pmed.1000097.

Morell-Mengual, V. et al. (2016). Sexualidad y abuso sexual en hombres y mujeres con discapacidad intelectual. En J. L. Castejón Costa (Coord.), Psicología y Educación: Presente y Futuro. ACIPE-Asociación Científica de Piscología y Educación.

Nosek, A. M. et al. (2001). Vulnerabilities for Abuse Among Women with Disabilities. Sexuality and Disability, 19, pp. 177-189. https://doi.org/10.1023/A:1013152530758.

Recio, M. et al. (2013). Abuso y Discapacidad Intelectual: orientaciones para la prevención y la actuación. Fundación Carmen Pardo-Valcarce.

Reiter, S. et al. (2007). Adolescents with intellectual disabilities as victims of abuse. Journal of Intellectual Disabilities, 11, pp. 371-387. https://doi.org/10.1177/1744629507084602.

Scotti, J. R. et al. (1996). College student attitudes concerning the sexuality of persons with mental retardation: Development of the Perceptions of Sexuality Scale. Sexuality and Disability, 14, pp. 249-263. https://doi. org/10.1007/BF02590098. 
Sobsey, D. y Varnhagen, C. (1988). Sexual abuse and exploitation of people with disabilities. Health and Welfare Canada.

Spencer, N. et al. (2005). Disabling conditions and registration for child abuse and neglect: a population-based study. Paediatrics, 116, pp. 609-613. https://doi.org/10.1542/peds.2004-1882.

Stromness, D. D. (1994). Sexually abused women with mental retardation: Hidden victims, absent resources. Women and Therapy, 14, pp. 139-152. https://doi.org/10.1300/J015v14n03_14.

Subdirección General de Planificación, Ordenación y Evaluación (13 de noviembre de 2020). Base estatal de datos de personas con valoración del grado de discapacidad. Informe a 31/12/2018. Imserso. https://www.imserso. es/imserso_01/documentacion/estadisticas/bd_estatal_pcd/index.htm.

Verdugo, M. A. et al. (2002). El abuso sexual en personas con discapacidad intelectual. Psicothema, 14, pp. 124129.

Viñuela, L. (2009). Mujeres con discapacidad: un reto para la teoría feminista. Feminismo/s, 13, pp. 33-48. https:// doi.org/10.14198/fem.2009.13.03.

Wigham, S. et al. (2011). The effects of traumatizing life events on people with intellectual disabilities: a systematic review. Journal of Mental Health Research in Intellectual Disabilities, 4, pp. 19-39. https://doi.org/10.1080/193 15864.2010.534576.

Withers, P. y Morris, J. (2012). Sexual exploitation of people with intellectual disabilities. En E. Emerson et al. (Eds.), Clinical psychology and people with intellectual disabilities (pp. 273-292). John Wiley and Sons. 